\title{
Rotary Wireless Inductive Transmitter Powered by ZVS Resonant Convertor
}

\author{
Dimitar D. Arnaudov ${ }^{1}$, Nikolay D. Madzharov ${ }^{2}$, Nikolay L. Hinov ${ }^{1}$ \\ ${ }^{I}$ Department of Power Electronics, Faculty of Electronic Engineering and Technologies, \\ Technical University of Sofia, \\ 8 Kliment Ohridski Blvd., BG-1756 Sofia, Bulgaria \\ ${ }^{2}$ Department of Electronics, Faculty of Electrical Engineering and Electronics, \\ Technical University of Gabrovo, \\ 4 H. Dimitar, Gabrovo, 5300, Bulgaria \\ hinov@tu-sofia.bg
}

\begin{abstract}
In this work, an inductive wireless power transfer module powered by resonant converter is studied. The system consists of a zero voltage switching resonant converter and a rotary wireless power transmitter. The design of the rotary wireless power transmitter is presented. Simulation and experimental studies of the system confirm reliable operation of the power electronic converter with a wide range of loads. The zero voltage switching is maintained without significant adjustments in the switching frequency. This improves the system stability with variations in the air gap between transmitting and receiving modules or the magnetic coupling ratio. The studied system is suitable for powering loads placed on a rotating platform or other applications where the disadvantages of moving electrical contacts are undesirable.
\end{abstract}

Index Terms-Inductive power transmission; Resonant inverter; Soft switching; Power conversion.

\section{INTRODUCTION}

In the recent years, wireless power has already become common in our everyday life and has the potential for numerous applications in the manufacturing and transportation sectors [1]. The development of this field of Power Electronics is also a result of the advances in manufacturing of power electronic switches and passive elements in the decades before. The optimized operation of the power electronic converters and newly developed ferromagnetic materials with improved characteristics lead to a reduction of losses, improved magnetic coupling, and efficiency of the conversion. The manufacturing of magnetodielectric materials allows for a variety of magnetic core and concentrator designs. All the above-mentioned opens new horizons for development of wireless power transmitters that can replace ordinary mechanical contact systems for supplying power to rotating parts, which could result in increased reliability and reduced maintenance of the manufacturing equipment.

Wireless transmitters are the basis for the production machines, conveyor, and production lines upgrade. It allows

Manuscript received 21 January, 2019; accepted 10 June, 2019.

The paper was supportedby the project "Model based design of power electronic devices with guaranteed parameters", ДН07/06/15.12.2016, Bulgarian National Scientific Fund. to supply power to loads in linear or rotational motion These loads are often mechanically fixed units that position and feed the workpiece into the machining tool or other type of module during the manufacturing process. Once the operation is complete, the workpiece is moved to the next manufacturing stage and a new one is positioned in its place. Thanks to the wireless transmission of power [1], [2], the positioning and feeding stands could keep their continuous movement during the production cycle. Their design is determined by the type of a workpiece and the machining steps. Mostly, they perform the following actions: catching a detail from a conveyor belt, directing and positioning, feeding a detail to a processing tool, deriving a detail from operation, and transmitting it to an output conveyor belt. The system described above can be constructed in two ways: via a linear or rotating motion of the positioning stands.

The simultaneous processing of a large number of operations requires synchronization between the positioning stand units. Sensors and limiters are used to monitor their status and position in order to keep them synchronized. There are also feedback signals from encoders for start, stop, overload, etc. Some of these signals have to be exchanged between the transmitter and the receiver controllers [3]-[5], which involves also wireless transmission of data and control signals.

A cross-section of a Rotating Platform (RP) is depicted in Fig. 1, which illustrates its structure and principle of operation. The driving motors and pneumatics, part of the synchronizing sensors common to all positioning stands, and a Rotary Wireless Transformer (RWT) are also shown.

Wireless power transmission impose a number of requirements for the design of high-frequency power supplies (most often resonant converters) due to the air gap and the movement of the transmitter and receiver. This results in weak coupling and increase of the leakage inductance [6]-[8]. Besides, a change in the resonant circuit parameters induce a change in the resonant frequency [9]. The Zero Voltage Switching (ZVS) operation of the circuit demands precise regulation of the switching frequency in order to maintain stable output characteristics of the converter [10], [11]. 


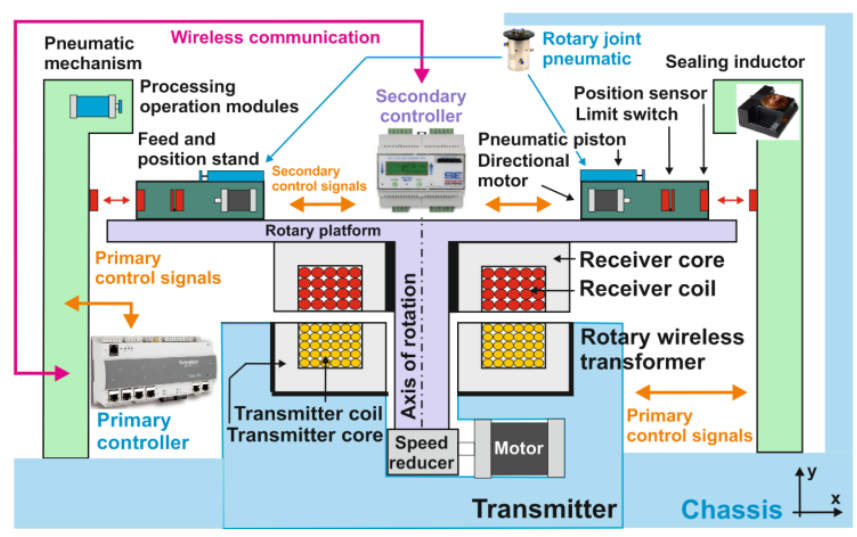

Fig. 1. Cross-section of a rotating platform.

RWT is the module that provides power to consumers situated in the RP. Ripple and voltage drops have to be avoided in order to ensure a reliable power for the loads. Nevertheless, efficiency is also a concern in wireless energy transfer. For this purpose, the precise design and analysis in various operating modes of the RWT and the power electronic converters are required, taking into account the size of the air gap and the positioning between the transmissions and receiving parts [12]-[16].

Subject to this study is a wireless rotating transformer powered by a zero-voltage switching resonant converter with improved characteristics designed for this particular application.

\section{ROTARY WIRELESS POWER TRANSMITTER}

\section{A. ZVS Power Converter}

A Zero Voltage Switching (ZVS) resonant converter is used in the studied contactless power supply system. The resonant converter power circuit is presented in Fig. 2.

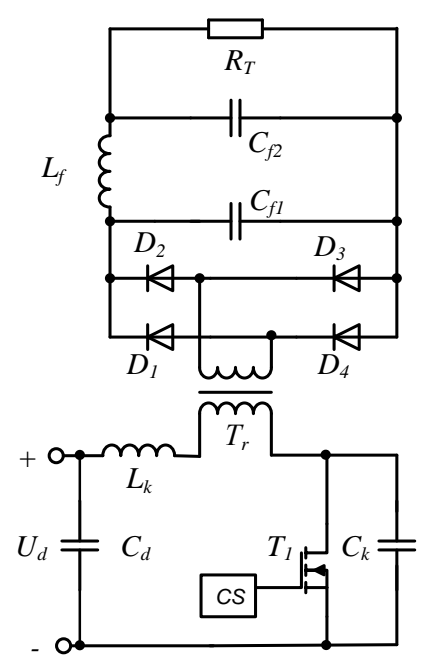

Fig. 2. ZVS resonant converter power circuit.

The primary winding of the transformer for wireless energy transmission $T_{r}$ is connected to the alternating current circuit of a series resonant inverter. The MOSFET $T_{l}$ has a body diode, which is not depicted in the figure. The operation of this circuit by ZVS and zero current switching is studied in numerous sources, including [10], [11]. A bridge rectifier with CLC filter is connected to the secondary winding of the transformer [17]. The circuit design for achieving ZVS operation and high performance of the converter within a broad range of loads are detailed in [10]. In this work, the resonant converter is used to power a rotary wireless transmitter.

\section{B. Design of RWT and Electromagnetic Processes}

There are two basic RWT structures - axial (A) and radial (R) [1], [12], [18].

The cross-section of the radial rotary transformer used in the construction of the RP is shown in Fig. 3. In this figure, the main geometric parameters of the RWT are depicted.

\section{Rotary platform}

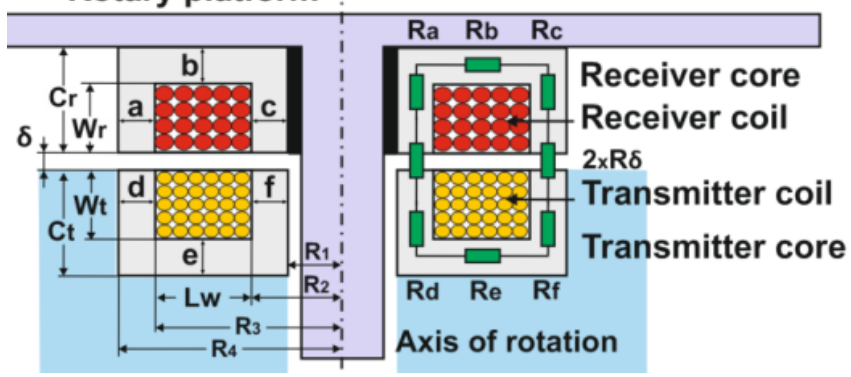

Fig. 3. Electromagnetic circuit of radial RWT.

The main design symbols and dimensions are as follows: $L_{w}$ is the length of both coils and $W_{r}, W_{t}$ are the widths of the receiver and transmitter coils. The transmitter and receiver widths are $C_{r}$ and $C_{t}$, respectively. $R_{l}$ is the internal radius of the RWT fixed to the diameter of the rotation shaft, $R_{2}, R_{3}$ are radiuses defining the width of the RWT coils, $R_{4}$ is the external radius of the RWT, a, b, c, d, e, and $\mathrm{f}$ are the geometric dimensions of the respective parts of the core, $\delta$ is an air gap, and b, e define the minimal section of the receiver and the transmitter.

By optimization of the $L_{w}, W_{r}(t)$, and $C_{r}(t)$ geometric dimensions, an improvement of the magnetic coupling between both coils and reduction of the parasitic parameters can be achieved [7], [8], [15], [16].

With $R$ the magnetic reluctances of the RWT chassis parts are denoted. They form a magnetic circuit with lengths $l(n)$ as the intensity $H$ of the field through them is constant. The sum of the individual lengths gives the average length of the magnetic core

$$
l=\sum l_{(n)}=l_{a}+l_{b}+l_{c}+l_{d}+l_{e}+l_{f}+2 l_{\delta}
$$

The "L" shaped equivalent circuit is used for the modelling of the transformer (Fig. 4).

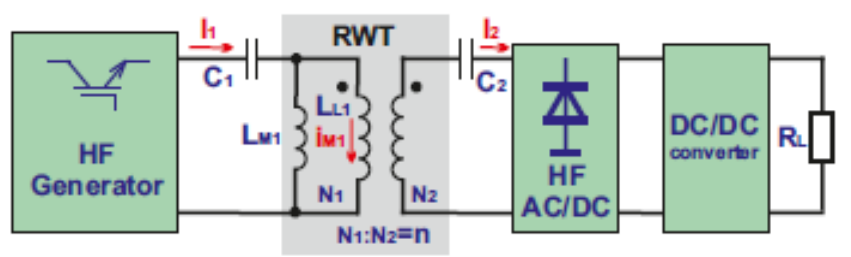

Fig. 4. Equivalent circuit of RWT system.

The electromagnetic energy stored in a volume $\mathrm{V}$ comprising the RWT core, air gar, the primary winding leakage inductance $L_{L l}$, and the magnetizing inductance $L_{M l}$, 
is expressed by

$$
W_{m}=\int_{v} H B / 2 d v=\mu_{0} H^{2} V / 2=L_{L 1} I^{2} / 2 .
$$

The field in of the transmitting and receiving coils for the distance $x\left(R_{2}<x<R_{3}-\right.$ Fig. 5) and the field strength in the air gap are defined by the following expressions:

$$
\begin{gathered}
H_{t}=\frac{N_{1} I_{1}}{R_{3}-R_{2}} \times \frac{x}{W_{t}}, \\
H_{r}=\frac{N_{2} I_{2}}{R_{3}-R_{2}} \times \frac{x}{W_{r}}, \\
H_{\delta}=N_{1} I_{1} / \delta,
\end{gathered}
$$

where $I_{1}, I_{2}$ are the currents through the transmitting and the receiving coils, $N_{1}$ and $N_{2}$, respectively, are the number of turns of the transmitting and receiving windings.

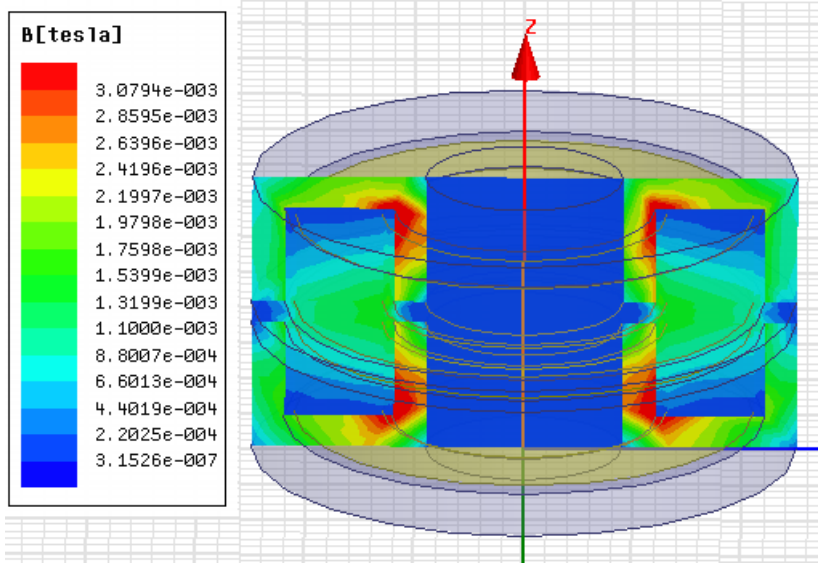

Fig. 6. Simulation results of the flux density in the radial RWT.

Using (2) and (3)-(5), the following for the average value of the field strength of the transmitting coil becomes as

$$
H_{t}=\frac{N_{1} \cdot I_{1}}{R_{3}-R_{2}} \times \frac{1}{\sqrt{W_{t}}} \int_{0}^{W_{r t}} \frac{x}{W_{t}} d z=\frac{N_{1} I_{1}}{R_{3}-R_{2}} \times \frac{1}{\sqrt{3}} .
$$

The expressions for the receiving coil and the air gap field strength are obtained analogically. The energy $W_{m}$, stored in the RWT magnetic circuit is expressed by

$$
W_{m}=\frac{1}{2} \mu_{0} \times \frac{N_{1}^{2} I_{1}^{2} \pi}{\left(R_{3}-R_{2}\right)^{2}}\left(R_{3}+R_{2}\right) \times\left[\frac{W_{t}}{3}+\frac{W_{r}}{3}+\delta\right] \text {. }
$$

From (2)-(7), the expression for the leakage inductance $L_{L l}$ becomes as follows

$$
L_{L 1}=\mu_{0} N_{1}^{2} \times \frac{\pi\left(R_{3}+R_{2}\right)}{R_{3}-R_{2}} \times\left(\delta+\frac{W_{t}+W_{r}}{3}\right) .
$$

The equations describing the equivalent magnetic circuit are used to express the magnetizing inductance. It consists of the magnetic reluctances of the core and the air gap. The equivalent magnetic reluctance of the equivalent circuit is

$$
R_{\Sigma}=R_{e}+R_{b}+R_{f}+R_{c}+R_{d}+R_{a}+2 R_{\delta}
$$

and

$$
R_{(n)}=l_{(n)} / \mu_{M} S_{(n)},
$$

where $l(n)$ and $S(n)$ are the length and the ? is the cross sectional area of the respective part; $\mu_{M}$ is the magnetic permeability of the material. From (10) and Fig. 4 the magnetizing inductance is expressed as:

$$
L_{M 1}=N_{1}^{2} / R_{\Sigma}
$$

The currents flowing through the transmitter and receiver coils generate the magnetic fluxes $\Phi_{1}$ and $\Phi_{2}$ as depicted in Fig. 6.

The main flux generated by the currents flowing through both coils $\Phi_{0}=\Phi_{1}+\Phi_{2}$ is passing through the magnetic core and the flux generated by the leakage inductances of the coils, which are $\Phi_{\sigma}=\Phi_{\sigma 1}+\Phi_{\sigma 2}$. As a result, the flux in the RWT is expressed by [12]

$$
\Phi=\Phi_{0}+\Phi_{\sigma}
$$

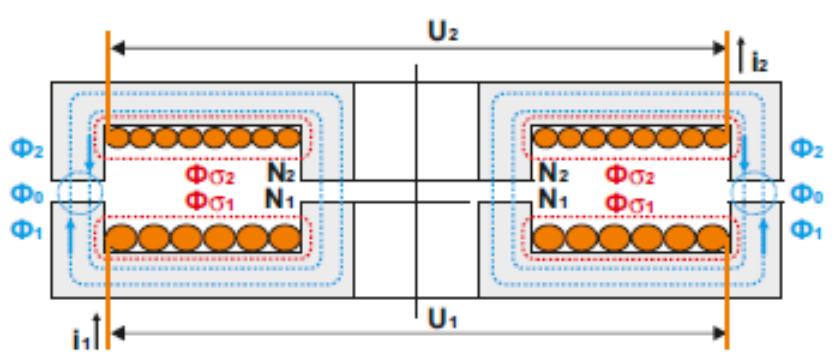

Fig. 6. Magnetic flux in the RWT.

The value of the magnetic field $F_{m}$ is obtained from the following expressions:

$$
\begin{gathered}
F_{m}=N_{1} i_{1}, \\
F_{m}=\Phi R_{\Sigma},
\end{gathered}
$$

where $i_{1}$ is the current flowing through the transmitter coil [2], [8]-[10].

This magnetic circuit of the RWT is described by the following expressions

$$
\begin{aligned}
F_{m} & =\Phi R_{\Sigma}=\Phi \times\left(R_{M}+R_{\delta}\right)= \\
& =\Phi \times\left(\frac{l_{(n)}}{\mu_{M} S_{(n)}}+\frac{l_{\delta}}{\mu_{0} S}\right) .
\end{aligned}
$$

From (13)-(15), numbers of turns of the primary and secondary coils are expressed:

$$
\begin{aligned}
& N_{1}=\frac{U_{E}}{S_{\text {min }} \Delta B f}, \\
& N_{2}=\frac{U_{\text {out }}}{U_{E}} \times N_{1},
\end{aligned}
$$

where $S_{\min }$ is the minimum cross-sectional area of the magnetic core, $\Delta B$ is the flux density, $U_{E}$ is the power supply voltage, $f$ is the working frequency, and $U_{\text {out }}$ is the output 
voltage.

The simulation results of the designed radial RWT are presented in Fig. 5. The analysis of these results shows that the designed core also shields the field and satisfies the electromagnetic compatibility criteria due to the flux density outside its magnetic core is less than $15 \mu \mathrm{T}$. The unequally distributed flux density is stronger in the direction parallel to the rotational axis $(2.63 \mathrm{mT}-3.07 \mathrm{mT})$. According to existing standards for electromagnetic field dissipation, the approved safety levels for frequencies up to $150 \mathrm{kHz}$ are $\mathrm{B}=$ $27 \mu \mathrm{T}$.

\section{SimUlation AND EXPERIMENTAL RESUlTS}

The electromagnetic parameters of the RWT are strongly dependent of to the coupling ratio $k$, and thus of the air gap between both windings. In order to test the design, experimental studies are conducted with the developed RWT prototype presented in Fig. 7. The inner and outer radius $\left(R_{2}\right.$ and $R_{3}$ in Fig. 3 ) of the transmitting and receiving windings are $20 \mathrm{~mm}$ and $75 \mathrm{~mm}$, respectively. During the design stage, the magnetic coupling ratio is being calculated for air gap values in the range from $2 \mathrm{~mm}$ to $20 \mathrm{~mm}$ [2].

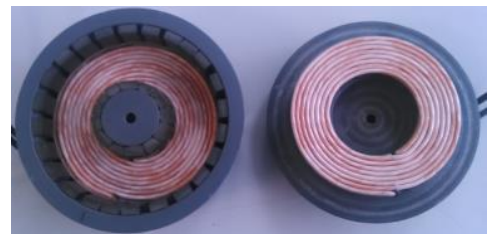

(a)

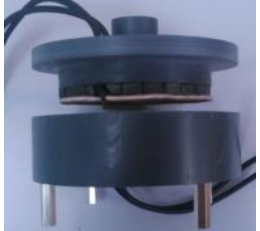

(b)
Fig. 7. The wireless transformer (a), (b) used in the experimental study.

In Table I, the experimental results with an active load in the receiver coil are presented. The active power and the efficiency are calculated by the following formulae:

$$
P=U I \cos \varphi,
$$

and

$$
\eta=P_{2} / P_{1}
$$

where $\cos \varphi$ is the power factor, $P_{1}$ and $P_{2}$ are the active powers in the transmitter, and receiver coils, respectively.

TABLE I. RESULTS FROM THE EXPERIMENTAL STUDY OF THE RWT WITH VARIOUS AIR GAPS FROM 2 MM TO $20 \mathrm{MM}$.

\begin{tabular}{|c|c|c|c|c|c|c|c|}
\hline Air gap [mm] & $\mathbf{2 0}$ & $\mathbf{1 5}$ & $\mathbf{1 2}$ & $\mathbf{9}$ & $\mathbf{6}$ & $\mathbf{4}$ & $\mathbf{2}$ \\
\hline $\mathbf{k}$ & 0,2 & 0,3 & 0,4 & 0,5 & 0,6 & 0,7 & 0,8 \\
\hline $\mathbf{U}_{\mathbf{1}},[\mathbf{V}]$ & 196 & 187 & 168 & 144 & 101 & 84 & 120 \\
\hline $\mathbf{I}_{\mathbf{1}},[\mathbf{A}]$ & 24 & 22 & 20 & 16 & 11 & 9 & 13 \\
\hline $\boldsymbol{\varphi},\left[{ }^{\circ}\right]$ & 86,6 & 86,2 & 85,4 & 84,6 & 82,1 & 79,4 & 83,3 \\
\hline $\mathbf{P}_{\mathbf{1}},[\mathbf{W}]$ & 279 & 273 & 269 & 216 & 153 & 139 & 168 \\
\hline $\mathbf{U}_{\mathbf{2}},[\mathbf{V}]$ & 32 & 46 & 55 & 59 & 52 & 50 & 57 \\
\hline $\mathbf{I}_{\mathbf{2}},[\mathbf{A}]$ & 1,59 & 2,28 & 2,75 & 2,95 & 2,72 & 2,56 & 2,84 \\
\hline $\mathbf{P}_{\mathbf{2 t}},[\mathbf{W}]$ & 51 & 105 & 151 & 174 & 141 & 128 & 161 \\
\hline $\boldsymbol{\eta},[\mathbf{\%}]$ & 18 & 38 & 56 & 81 & 92 & 92 & 96 \\
\hline
\end{tabular}

According to the presented experimental results, maximum efficiency is achieved by values of the coupling ratio $k$ between 0.6 and 0.8 . Values of the coupling ratio lower than 0.2 lead to a significant decrease in the efficiency. These coupling ratios correspond to air gaps values larger than $20 \mathrm{~mm}$ and ratio of the air gap and transmitter coil diameter higher than 0.25 . According to the results, the average efficiency of RWT is $94 \%$ at $500 \mathrm{~W}$ of power transferred to the load.

In order to study the ZVS converter operation, computer simulations and experimental tests are implemented. The values of the circuit parameters (Fig. 2) are: $U_{d}=60 \mathrm{~V}$, $L_{k}=75 \mu \mathrm{H}, \quad C_{k}=0.15 \mu \mathrm{F}, \quad L_{l}=28.6 \mu \mathrm{H} \quad$ (primary $\quad$ RWT inductance), $L_{2}=6.8 \mu \mathrm{H}$ (secondary RWT inductance), $C_{f l}=100 \mu \mathrm{F}, C_{f 2}=10 \mu \mathrm{F}, L_{f}=100 \mu \mathrm{H}, R_{t}=2.2 \Omega, f=38.5 \mathrm{kHz}$ and the RWT magnetic coupling ratio is 0.8 . In Fig. 7, the RWT used in the experimental study is shown.

In Fig. 8, the simulation results of the power electronic converter are presented: $u_{T 1}$ is the voltage across the transistor $T_{l}$ and $i_{L k}$ is the current flowing through the inductance $L_{k}$.

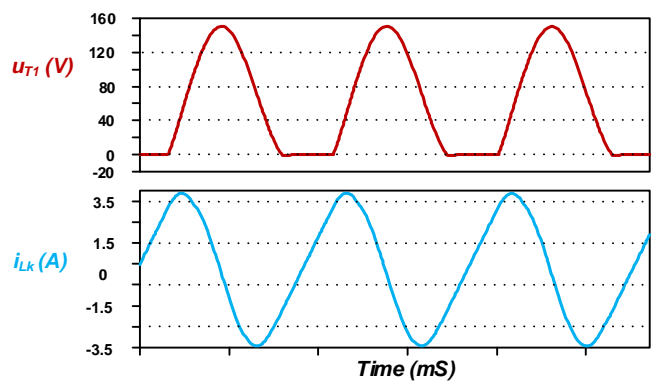

Fig. 8. Simulation results for the voltage across $T_{l}$ and current through $L_{k}$.

In Fig. 9, the experimental waveforms of $u_{T 1}$ (depicted in green) and $i_{L k}$ (blue) are presented. The current $i_{L k}$ is measured by the voltage across a shunt resistance of $0.1 \Omega$ (connected in series to $L_{k}$ ). From the comparison of these results, it is found that there is a very good concurrence (less than $5 \%$ difference).

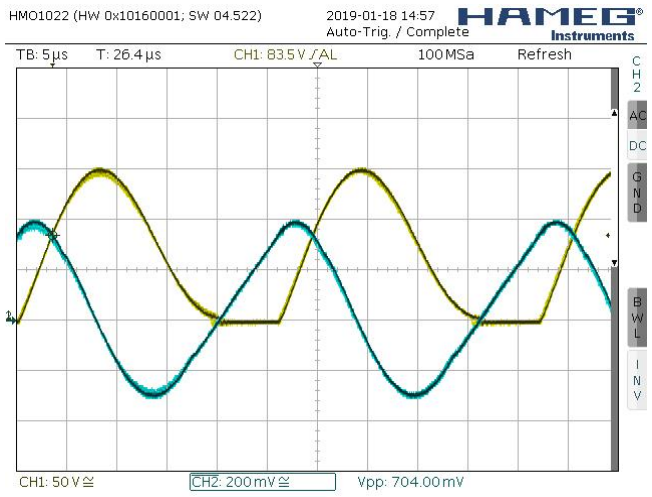

Fig. 9. Experimental results for voltage across $T_{1}$ (green) and current through $L_{k}$ (blue).

The waveforms of: the voltage across the primary RWT winding $u_{P R I M}$, the voltage across the secondary RWT winding $u_{S E C}$, the currents through diodes $D_{1}$ and $D_{2}-i_{D I}$ and $i_{D 2}$, respectively, and the voltage across the load $U_{0}$ are presented in Fig. 10-Fig. 12.

From the analysis of the results, it can be observed that $T_{l}$ peak voltage is around $160 \mathrm{~V}$, while the $U_{P R I M}$ voltage ripple is $16 \mathrm{~V}$ and the secondary $u_{S E C}$ is $6 \mathrm{~V}$. This is due to the fact that the output transformer is behaving like a current transformer; that is, the parameters have a weak influence on the resonant circuit. 


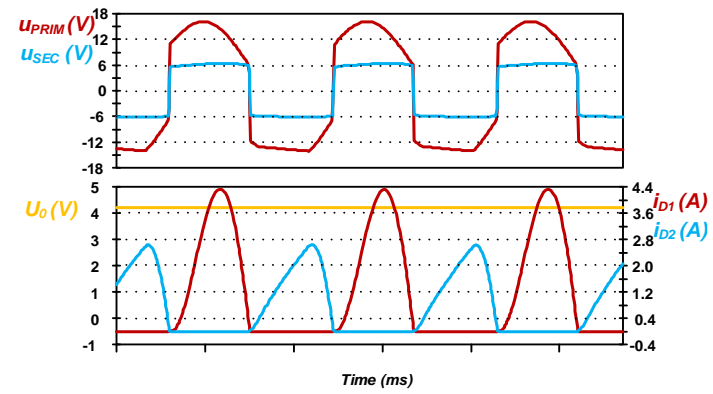

Fig. 10. Simulated waveforms of uPRIM, uSEC, iD1, iD2, and U0.

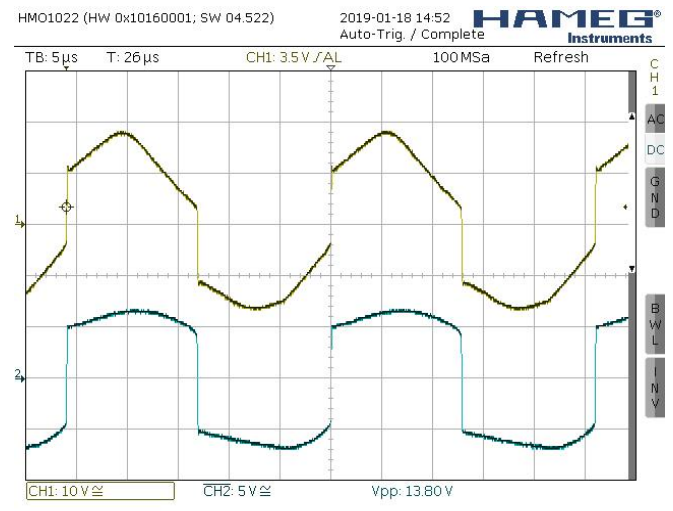

Fig. 11. Experimental waveforms of voltages across the primary (top) and secondary windings (bottom).

The difference between the simulated and measured values is less than $5 \%$, which confirms the proper design of the transformer and the ZVS resonant converter. The precision of the simulation results can be improved by a more detailed modelling of the RWT for contactless power transmission [6]-[8].

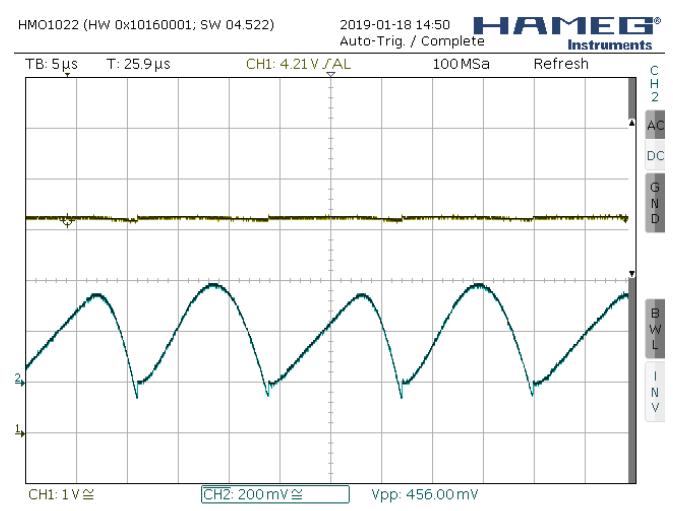

Fig. 12. Experimental results - voltage across the load (top) and the rectifier output current (bottom).

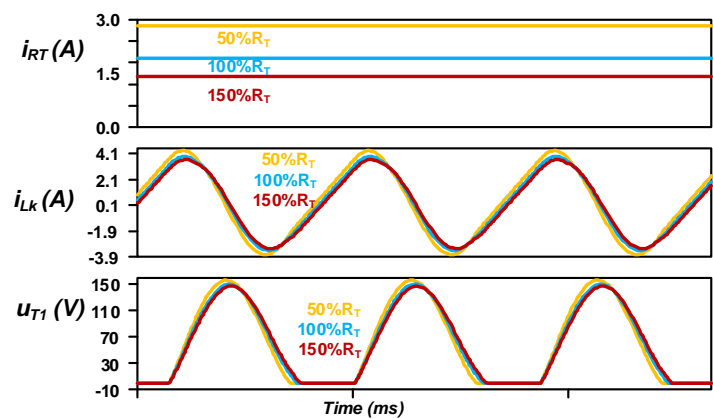

Fig. 13. Simulated waveforms of $i_{R T}, i_{L k}$, and $u T I$.

In Fig. 12, the waveform of the rectifier output current (before the CLC filter) measured with a $0.1 \Omega$ shunt resistor (connected between rectifier and CLC filter) is presented.

Figure 13 presents the results of a study of the operating mode of the converter at a load change in the range of $50 \%$ to $150 \%$ of the nominal value.

It can be seen that despite such a considerable change of load, the converter continues to operate in the ZVS mode.

\section{CONCLUSIONS}

A converter with contactless power transmission and ZVS is proposed. With presented basic ratios for the magnetic circuit, a rotary transformer is designed. To ensure ZVS operation, the implementation and appropriate tuning of the control system is necessary.

The simulation and experimental results confirm proper operation of the power electronic converter. ZVS operation is maintained over the range of variation of the load and magnetic coupling of the transformer.

The results demonstrate that the studied system is suitable for powering loads fixed on a platform rotating with high speed and charging energy storage elements, such as supercapacitors or batteries as well as other applications, in which significant variations in the load occur [11].

The presented design methodology, analysis of the prototype construction, and experimental study are a solid base for future design optimizations of RWT with numerous possible applications for inductive wireless power.

On the other hand, the implemented ZVS DC-DC converter with improved characteristics ensures stable and reliable operation of the system in a broad range of parameter fluctuations.

\section{REFERENCES}

[1] G. Covic and J. T. Boys, "Inductive power transfer", in Proc. of the IEEE, vol. 101, no. 6, pp. 1276-1289, 2013. DOI: 10.1109/JPROC.2013.2244536.

[2] M. Tosi, "Rotary transformer design for brushless electrically excited synchronous machines", M. S. thesis, University of Padova, Technische Universitat Munchen, 2014. [Online]. Available: http://tesi.cab.unipd.it/45556/1/Mastersthesis_To si_Mattia_1035471.pdf

[3] N. Bergmann, J. Juergens, L. Hou, Y. Wang, and J. Trevathan, "Wireless underwater power and data transfer", in Proc. 38th Annual IEEE Conference on Local Computer Networks - Workshops, pp. 21-24, 2013. DOI: 10.1109/LCNW.2013.6758505.

[4] W. Huang, B. Zhang, X. Chen, K.-M. Huang, and C.-J. Liu, "Study on an s-band rectenna array for wireless microwave power transmission", Progress In Electromagnetics Research, vol. 135, pp. 747-758, 2013. DOI: 10.2528/PIER12120314.

[5] M. Reddy, K. Hemanth, and C. Mohan, "Microwave power transmission a next generation power transmission system", IOSR Journal of Electrical and Electronics Engineering (IOSR-JEEE), vol. 4, no. 5, pp. 24-28, 2013. DOI: 10.9790/1676-0452428.

[6] R. Trevisan, and A. Costanzo, "A 1-kW contactless energy transfer system based on a rotary transformer for sealing rollers", IEEE Transactions on Industrial Electronics, vol. 61, no. 11, pp. 63376345, 2014. DOI: 10.1109/tie.2014.2311395.

[7] S. Ditze, A. Endruschat, T. Schriefer, A. Rosskopf, and T. Heckel, "Inductive power transfer system with a rotary transformer for contactless energy transfer on rotating applications", in Proc. 2016 IEEE International Symposium on Circuits and Systems (ISCAS), 2016. DOI: 10.1109/iscas.2016.7538876.

[8] J. Smeets, D. Krop, J. Jansen, and E. Lomonova, "Contactless power transfer to a rotating disk", in Proc. 2010 IEEE International Symposium on Industrial Electronics, 2010. DOI: 10.1109/isie.2010.5637414.

[9] G. Rizzoli, M. Mengoni, L. Zarri, and A. Tani, "Voltage feedback of 
an LLC resonant converter with a rotary transformer", in Proc. IECON 2018 - 44th Annual Conference of the IEEE Industrial Electronics Society, 2018. DOI: 10.1109/iecon.2018.8592682.

[10] T. Kitamoto, H. Omori, A. Murakami, T. Morizane, N. Kimura, and M. Nakaoka, "A novel type of high power-factor miniaturized wireless ev charger with optimized power receiving circuit and single-ended inverter", in Proc. IEEE International Power Electronics and Motion Control Conference (PEMC) 2016, Varna, 2016. DOI: 10.1109/EPEPEMC.2016.7752006.

[11] N. Hinov, D. Arnaudov, G. Kraev, and B. Gilev, "Modelling of single transistor parallel ZVS DC-DC converter", in Proc. of The 15th International Conference ELMA 2017, Sofia, 2017, pp. 158-162. DOI: 10.1109/ELMA.2017.7955422.

[12] N. Madzharov, V. Petkov, P. Kogias, and K. Karakoulidis, "Analysis of high-speed rotary wireless power transmitters", in Proc. of the XXVI International Scientific Conference Electronics (ET) 2017, 2017. DOI: 10.1109/ET.2017.8124363.

[13] D. Janulevicius, A. Valinevicius, D. Andriukaitis, "Intelligent plug-in hybrid electric vehicle", in Proc. of 7th international scientific conference Transport problems 2015, Katowice, Poland, 2015, pp. 215-226.

[14] N. Soni, R. Malekian, D. Andriukaitis, D. Navikas, "Internet of vehicles based approach for road safety applications using sensor technologies", “Wireless personal communications", vol. 105, iss. 4, pp. 1257-1284, 2019. DOI: 10.1007/s11277-019-06144-0.

[15] V. Valchev and A. Bossche, Inductors and transformers for power electronics. CRC Press Textbook, 2005.

[16] C. W. T. McLyman, Transformer and Inductor Design Handbook (Electrical and Computer Engineering) 4th Edition. CRC Press, 2011. DOI: doi.org/10.1201/b10865.

[17] N. Hinov and D. Arnaudov, Schemes of Single Transistor DC-DC Resonant Converters with Zero Voltage Switching and improved characteristics, Patent request for an invention 112869/2019, 2019.

[18] J. Smeets, L. Encica, and E. Lomonova, "Comparison of winding topologies in a pot core rotating transformer", in Proc. 12th International Conference on Optimization of Electrical and Electronic Equipment, 2010, pp. 103-110. DOI: 10.1109/OPTIM.2010.5510563. 